\title{
Commissioning of the ATLAS Reconstruction Software with First Data
}

\author{
Adam Gibson \\ University of Toronto \\ For the ATLAS Offline Commissioning Group and the \\ ATLAS Collaboration
}

IEEE NSS 2008: Dresden

October 22, 2008 


\section{ATLAS \\ Collaboration}

(Status July 2008)

\section{Countries \\ 169 Institutions \\ 2500 Scientific Authors total (1800 with a PhD)}

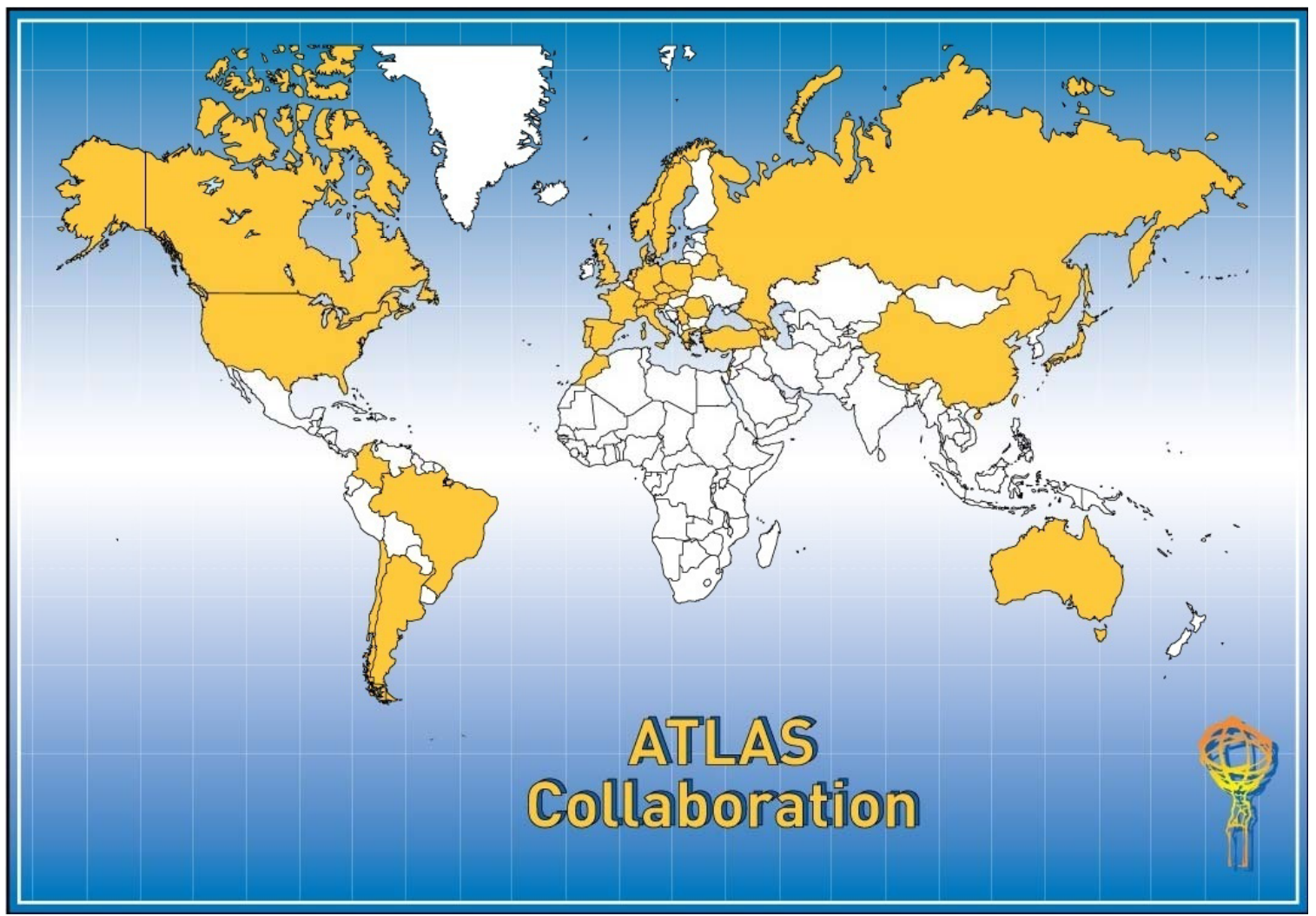

Albany, Alberta, NIKHEF Amsterdam, Ankara, LAPP Annecy, Argonne NL, Arizona, UT Arlington, Athens, NTU Athens, Baku, IFAE Barcelona, Belgrade, Bergen, Berkeley LBL and UC, HU Berlin, Bern, Birmingham, UAN Bogota, Bologna, Bonn, Boston, Brandeis, Bratislava/SAS Kosice, Brookhaven NL, Buenos Aires, Bucharest, Cambridge, Carleton, Casablanca/Rabat, CERN, Chinese Cluster, Chicago, Chile, Clermont-Ferrand, Columbia, NBI Copenhagen, Cosenza, AGH UST Cracow, IFJ PAN Cracow,

UT Dallas, DESY, Dortmund, TU Dresden, JINR Dubna, Duke, Frascati, Freiburg, Geneva, Genoa, Giessen, Glasgow, Göttingen, LPSC Grenoble, Technion Haifa, Hampton, Harvard, Heidelberg, Hiroshima, Hiroshima IT, Indiana, Innsbruck, Iowa SU, Irvine UC, Istanbul Bogazici, KEK, Kobe, Kyoto, Kyoto UE, Lancaster, UN La Plata, Lecce, Lisbon LIP, Liverpool, Ljubljana, QMW London, RHBNC London, UC London, Lund, UA Madrid, Mainz, Manchester, CPPM Marseille, Massachusetts, MIT, Melbourne, Michigan, Michigan SU, Milano, Minsk NAS, Minsk NCPHEP, Montreal, McGill Montreal, FIAN Moscow, ITEP Moscow, MEPhI Moscow,

MSU Moscow, Munich LMU, MPI Munich, Nagasaki IAS, Nagoya, Naples, New Mexico, New York, Nijmegen, BINP Novosibirsk, Ohio SU,

Okayama, Oklahoma, Oklahoma SU, Olomouc, Oregon, LAL Orsay, Osaka, Oslo, Oxford, Paris VI and VII, Pavia, Pennsylvania, Pisa,

Pittsburgh, CAS Prague, CU Prague, TU Prague, IHEP Protvino, Regina, Ritsumeikan, UFRJ Rio de Janeiro, Rome I, Rome II, Rome III, Rutherford Appleton Laboratory, DAPNIA Saclay, Santa Cruz UC, Sheffield, Shinshu, Siegen, Simon Fraser Burnaby, SLAC, Southern Methodist Dallas, NPI Petersburg, Stockholm, KTH Stockholm, Stony Brook, Sydney, AS Taipei, Tbilisi, Tel Aviv, Thessaloniki, Tokyo ICEPP, Tokyo MU, Toronto, TRIUMF, Tsukuba, Tufts, Udine/ICTP, Uppsala, Urbana UI, Valencia, UBC Vancouver, Victoria, Washington, Weizmann Rehovot, FH Wiener Neustadt, Wisconsin, Wuppertal, Würzburg, Yale, Yerevan 


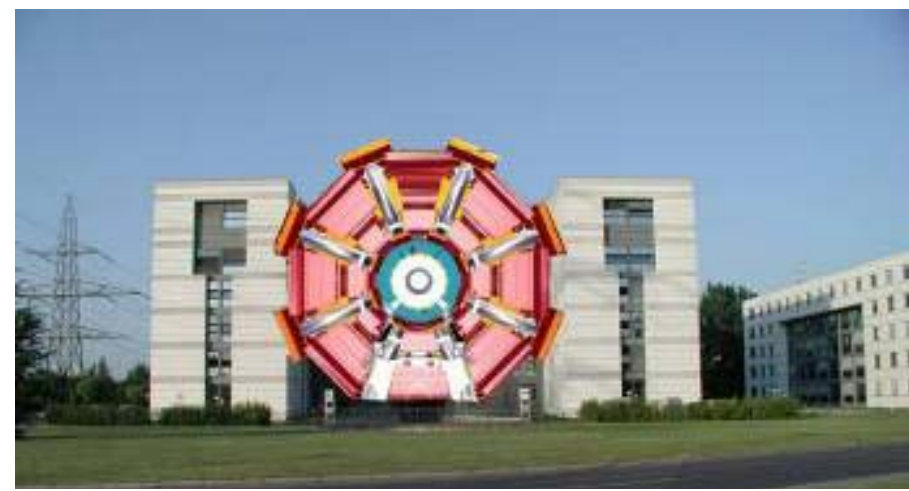

ATLAS

(A Toroidal LHC ApparatuS)

Detector
Length: 46 m

Radius: $\sim 12 \mathrm{~m}$

Weight: $~ 7000$ tons $\sim 10^{8}$ electronic channels

$\sim 3000 \mathrm{~km}$ of cables

ATLAS superimposed to the 5 floors of building 40

\section{$2 \mathrm{~T}$ central solenoid}

Air core toroidal magnets

Barrel and Endcap

Inner Detector (tracking)

Silicon Pixel

Silicon Strips (SCT)

Transition radiation tracker (TRT)

\section{Calorimeter}

EM LAr accordion

Hadronic LAr endcaps

Scintillating Tile

hadronic calorimeter

\section{Muon Spectrometer}

Monitored Drift Tubes (MDT)

Cathode Strip Chambers (CSC)

Resistive Plate Chambers (RPC)

Thin Gap Chambers (TGC)

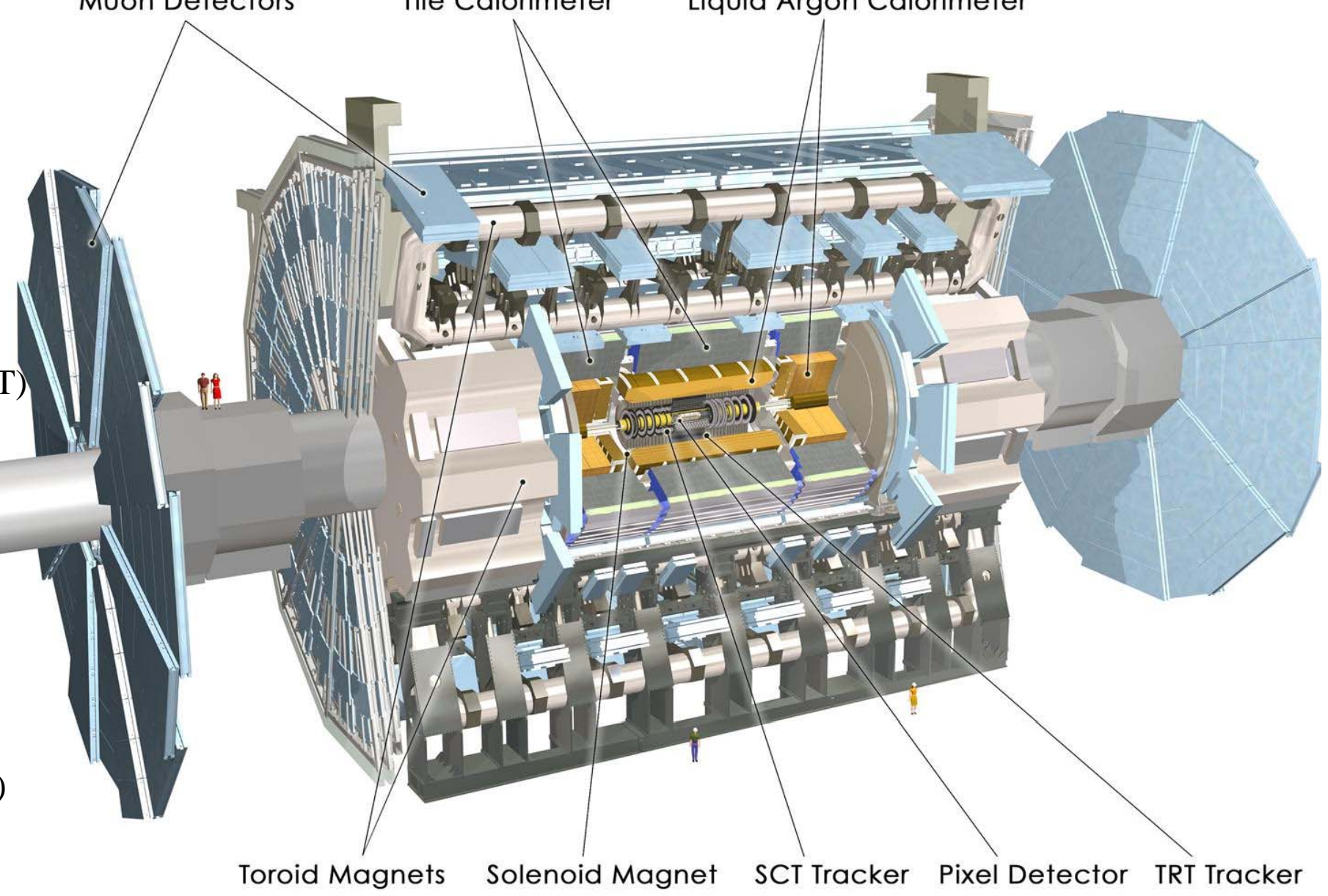




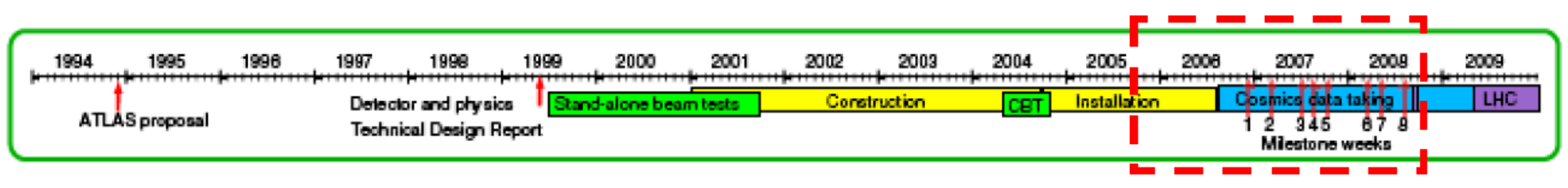

LHC

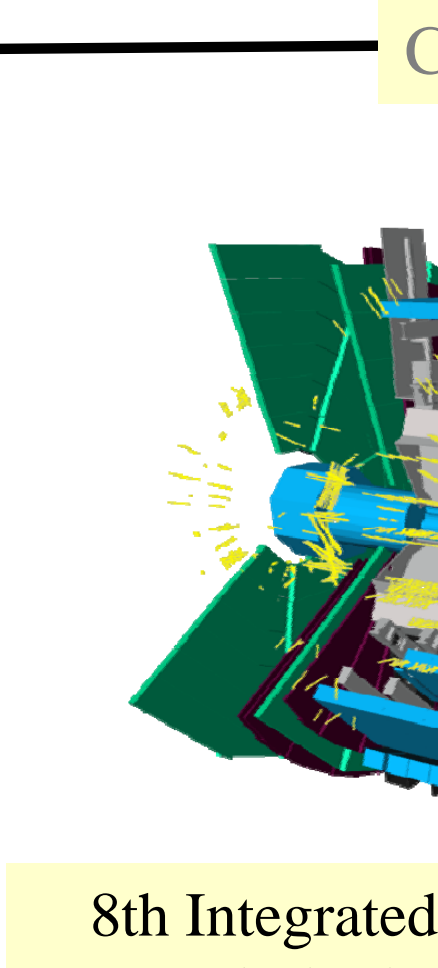

Commissioning

Period (M8)

11-20 July
Cosmic rays

single beam 10-12 Sept
Cosmic rays

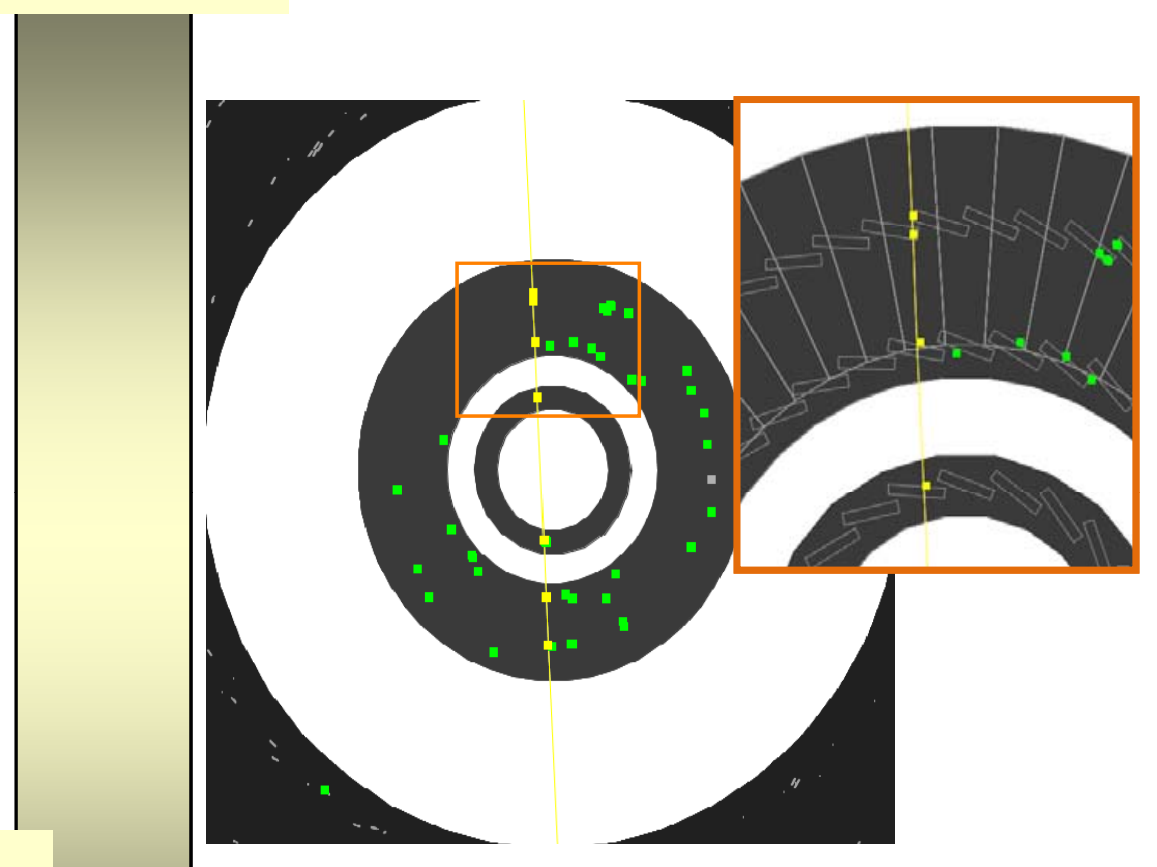

Pixels in 14th Sept
TRT Xe gas 


\section{ATLAS Reconstruction Framework}

- Athena (adapted from LHCb’s Gaudi) is the ATLAS software framework

- Used for

- High-level trigger

- Simulation

- Reconstruction

- Analysis and monitoring

- Runs at

- Online: High-level trigger, monitoring

- Tier-0: Prompt reconstruction

- Calibration centers: Feedback to T0, T1

- Tier-1: Reprocessing

- Tier-2, Tier-3 computing centers

- Adapts to commissioning environment

Simulation

Cosmic generator GEANT4

Digitization

Reconstruction

Integrate different subsystems

Combined algorithms

DB

Monitoring \& Analysis

Event display

Combined monitoring

Subsystem commissioning

- Converging on final ATLAS configuration

p. 5 


\section{Simulation for Commissioning}

- Unusual particle scenarios

- Cosmics

- Beam halo and beam gas from single beams

- Various detector configurations

- “Garaged” endcaps

- Misalignments

- With and without magnetic fields

- All impact how you generate, simulate, and digitize events, and how you reconstruct MC and real data

- e.g. A Cosmic Scenario

- Generate \pm 300 m, $10 \mathrm{GeV}$ - $5 \mathrm{TeV}$

- Simulate if pointing to sphere

- Rock overburden, with asymmetric access shafts

- Keep events only with simulated hit in detector
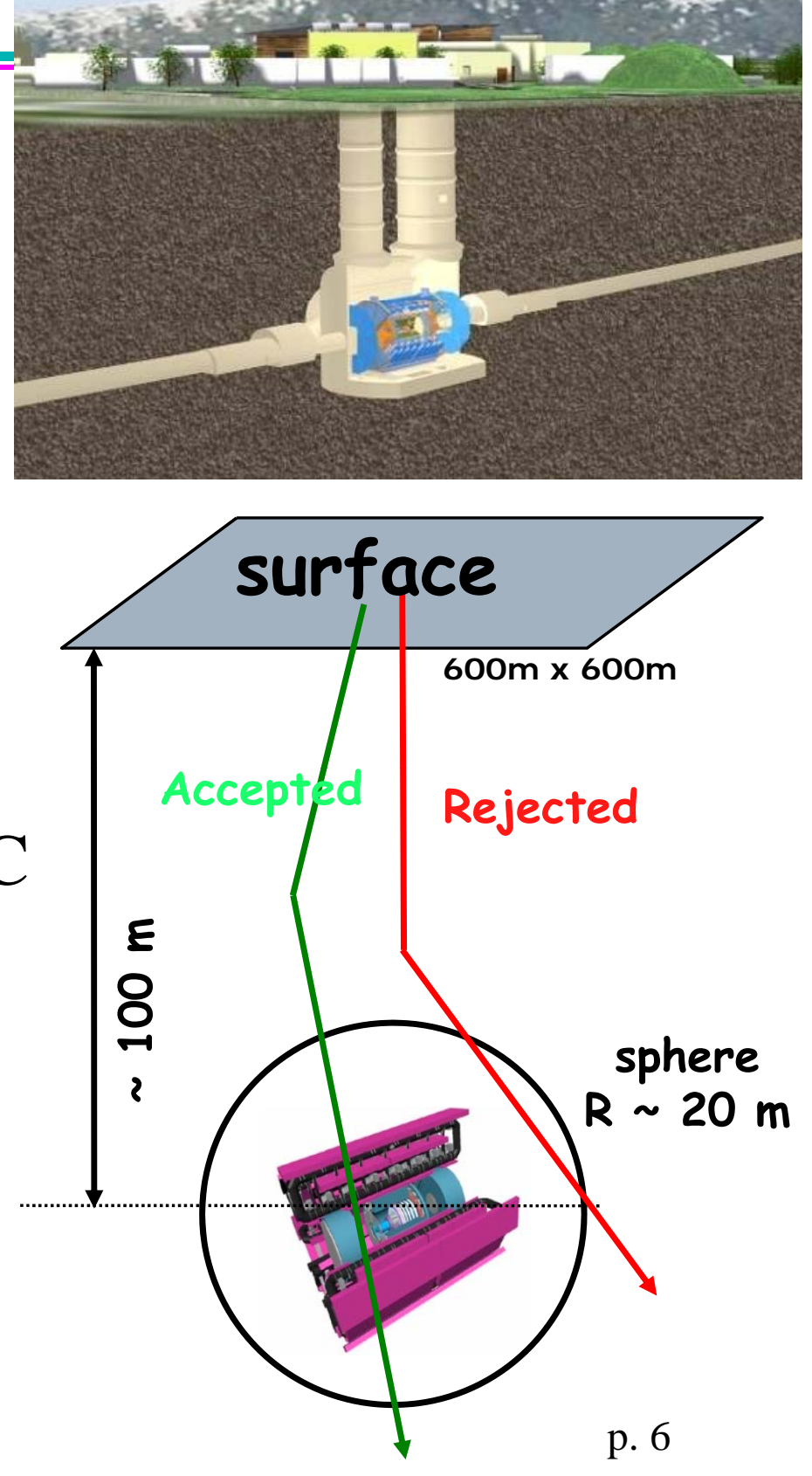


\section{Example of Cosmics Simulation: Muon tracks extrapolated to surface}

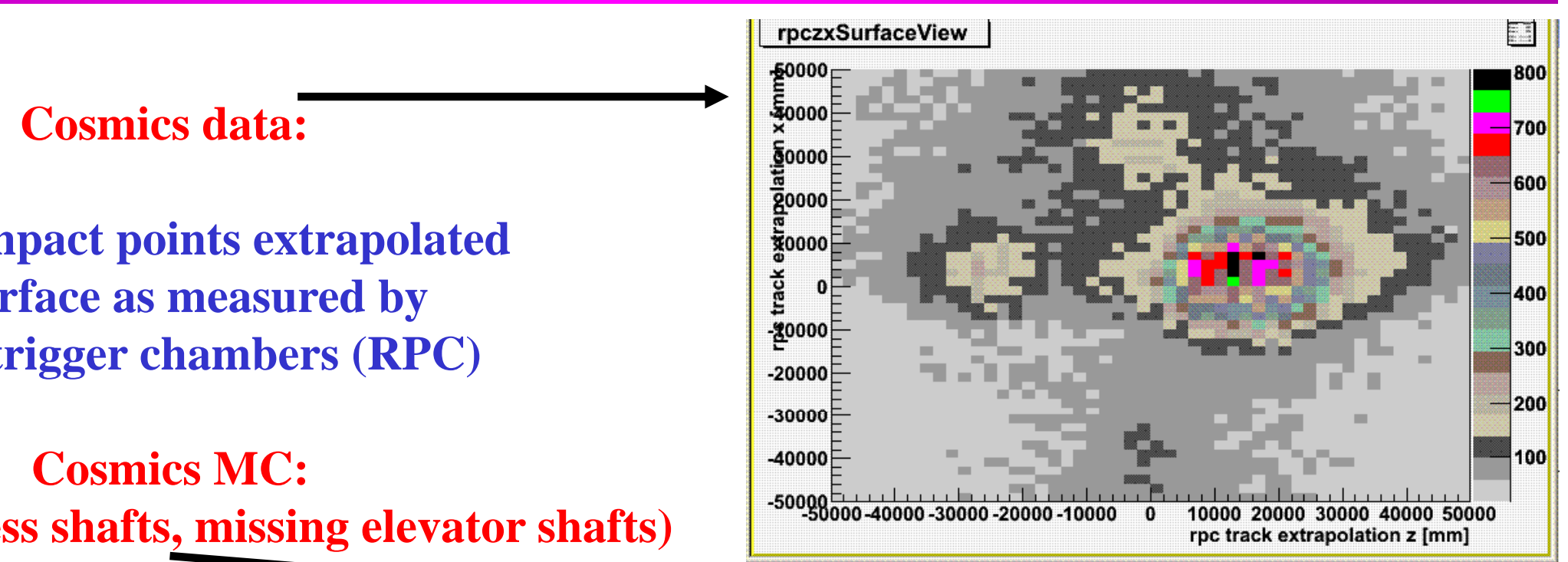

(With main access shafts, missing elevator shafts)

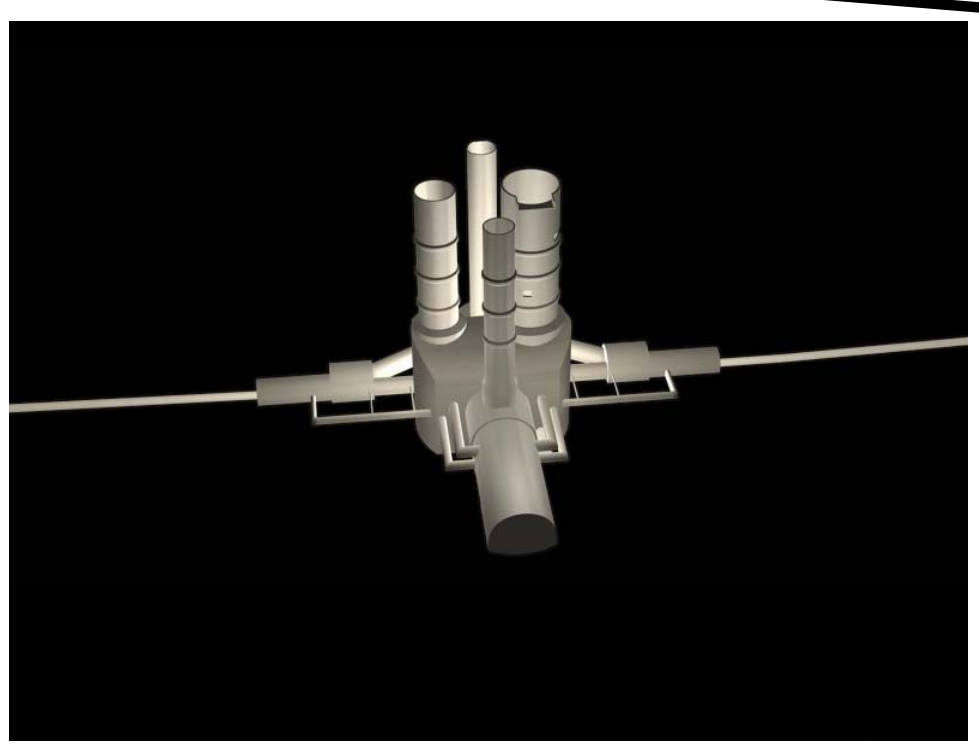

Reconstructed muon position at the surface

muons from small shaft muons from large shaft

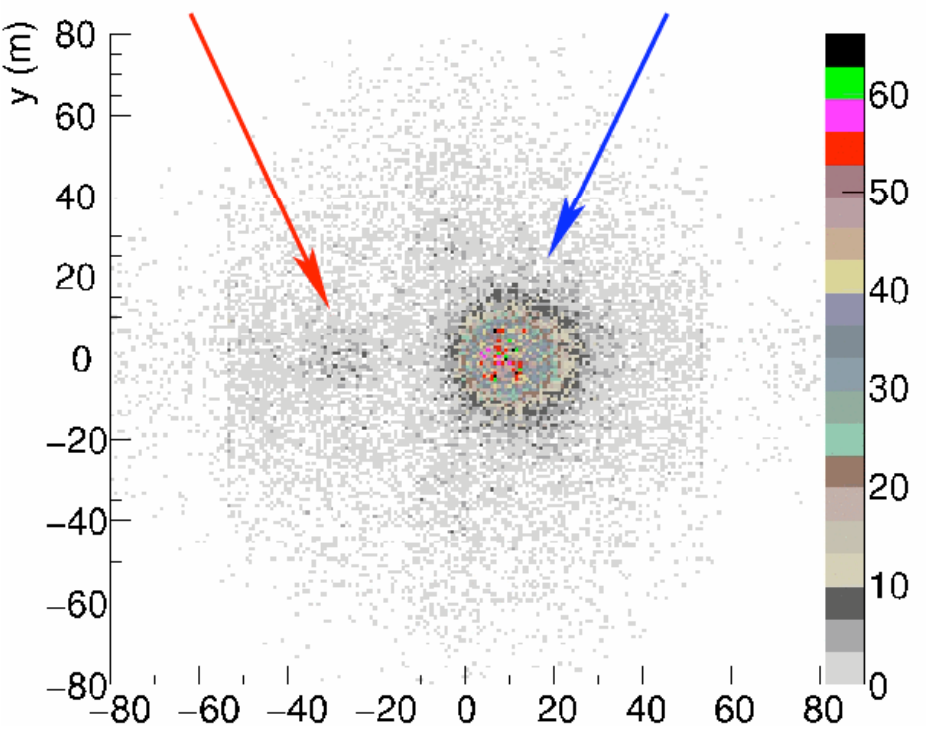

October 22, 2008 


\section{Online Reconstruction}

- Full reconstruction runs in the ATLAS control room

- Detector monitoring and data quality

- Event displays

- 2D and 3D displays, live feeds
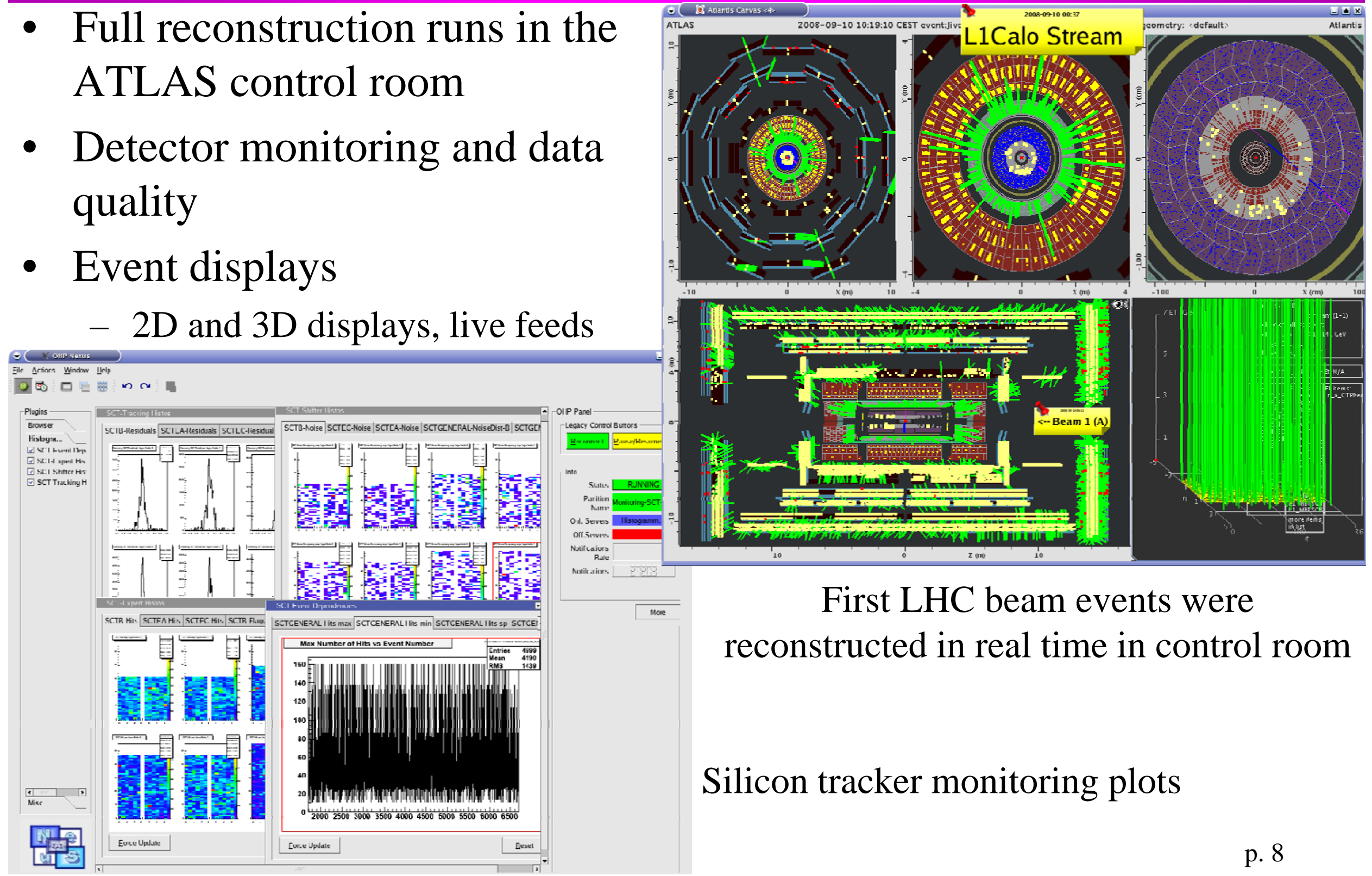

First LHC beam events were reconstructed in real time in control room

Silicon tracker monitoring plots 


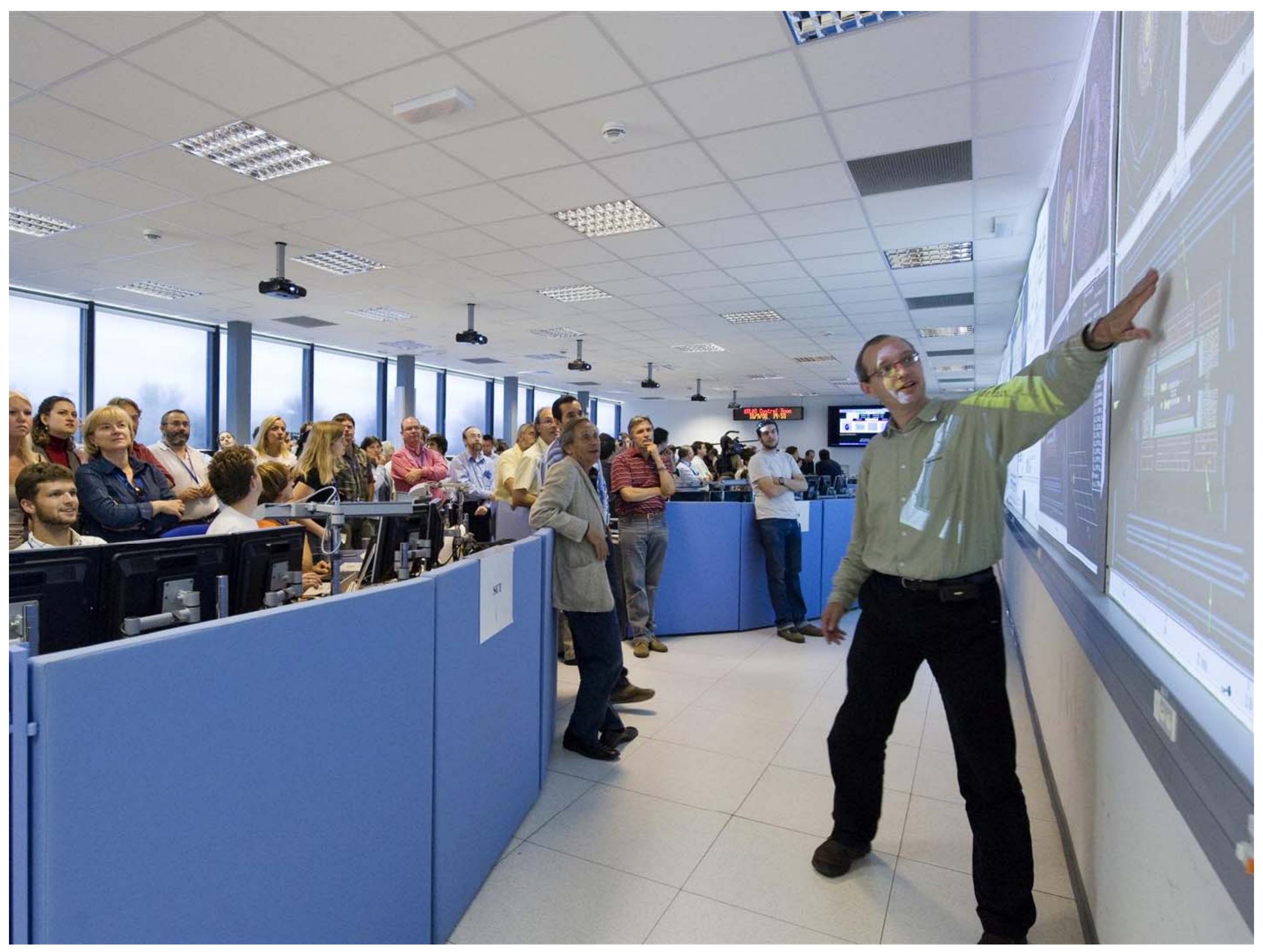




\section{Event Displays and Online Monitoring}

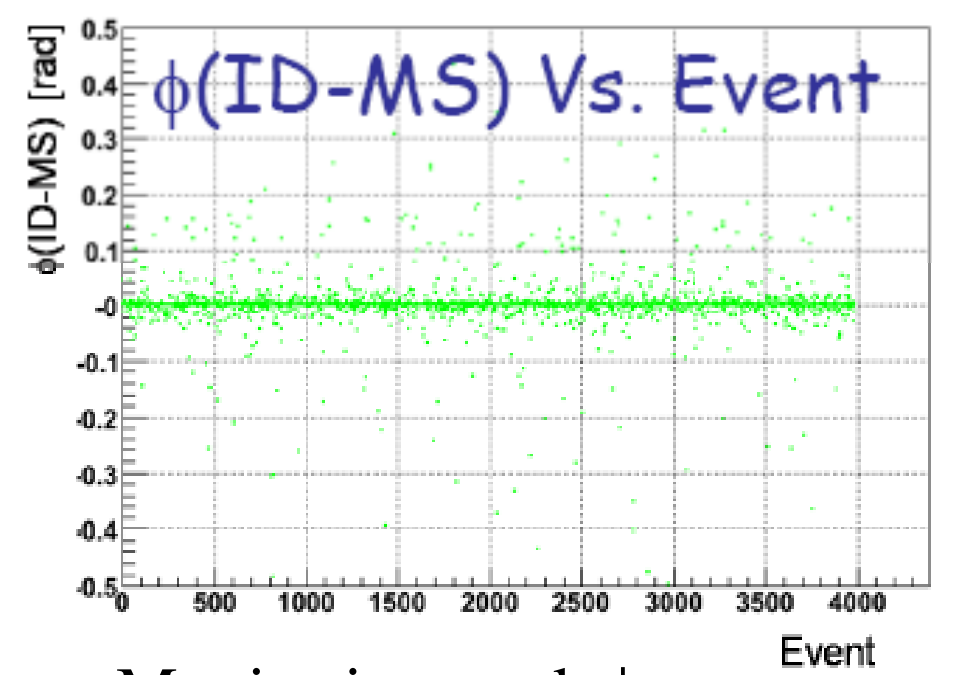

Monitoring track $\phi$ measured in the inner detector and muon spectrometer would e.g. allow you to see a loss of synchronization

Beam halo event from single LHC beam data

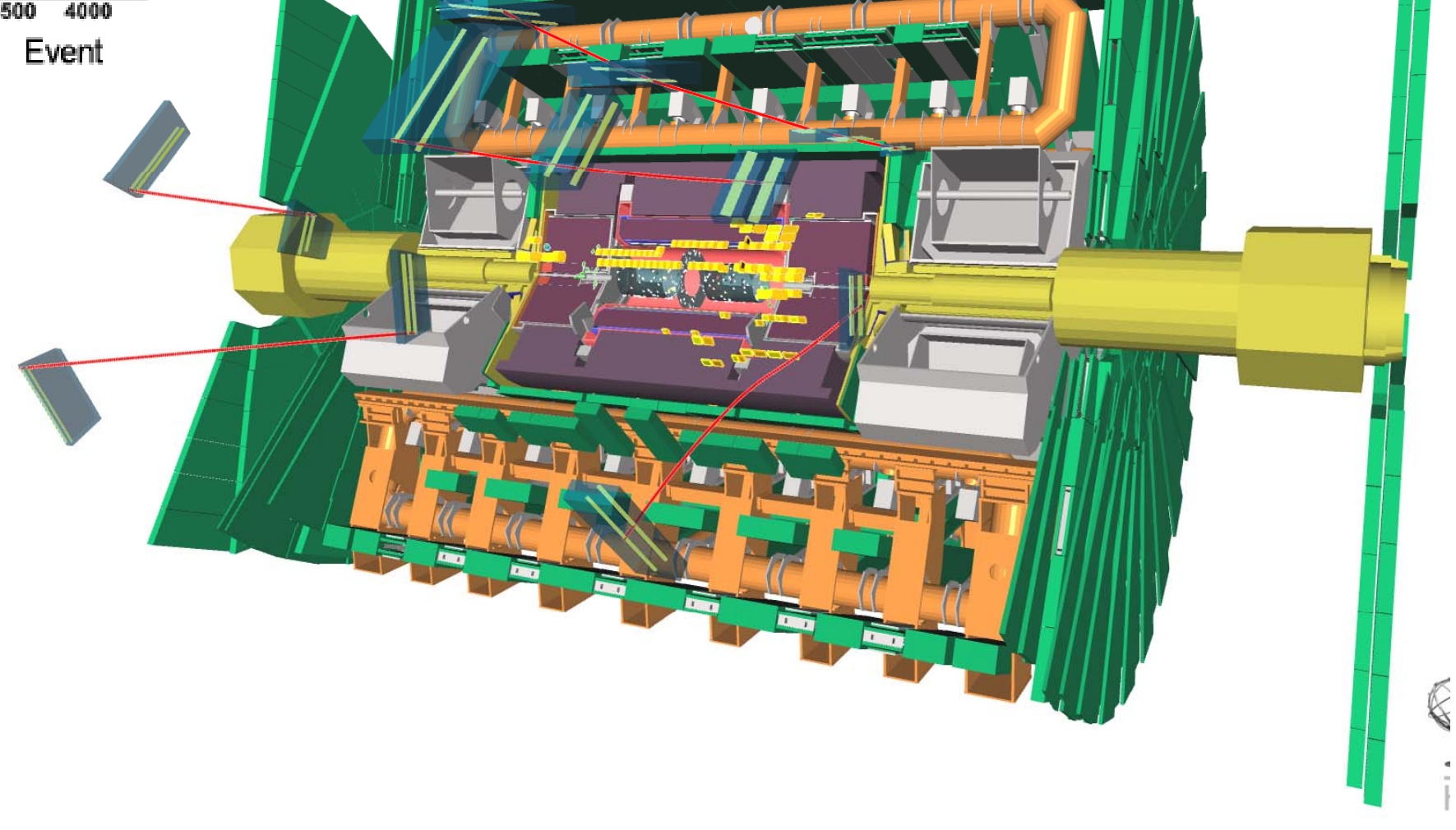




\section{Calibration and Alignment}

- Dedicated data/trigger streams for calibration and alignment

- Some subdetectors use dedicated calibration runs

- Dedicated computing facilities

- $\quad 1^{\text {st }}$ pass calibration and alignment within 24 hours

- Input to bulk reconstruction at Tier-0

- "Best effort” calibration and alignment can be applied to reprocessing at Tier-1 centers

- Time scale $\sim$ months

- Tested extensively with cosmics and MC
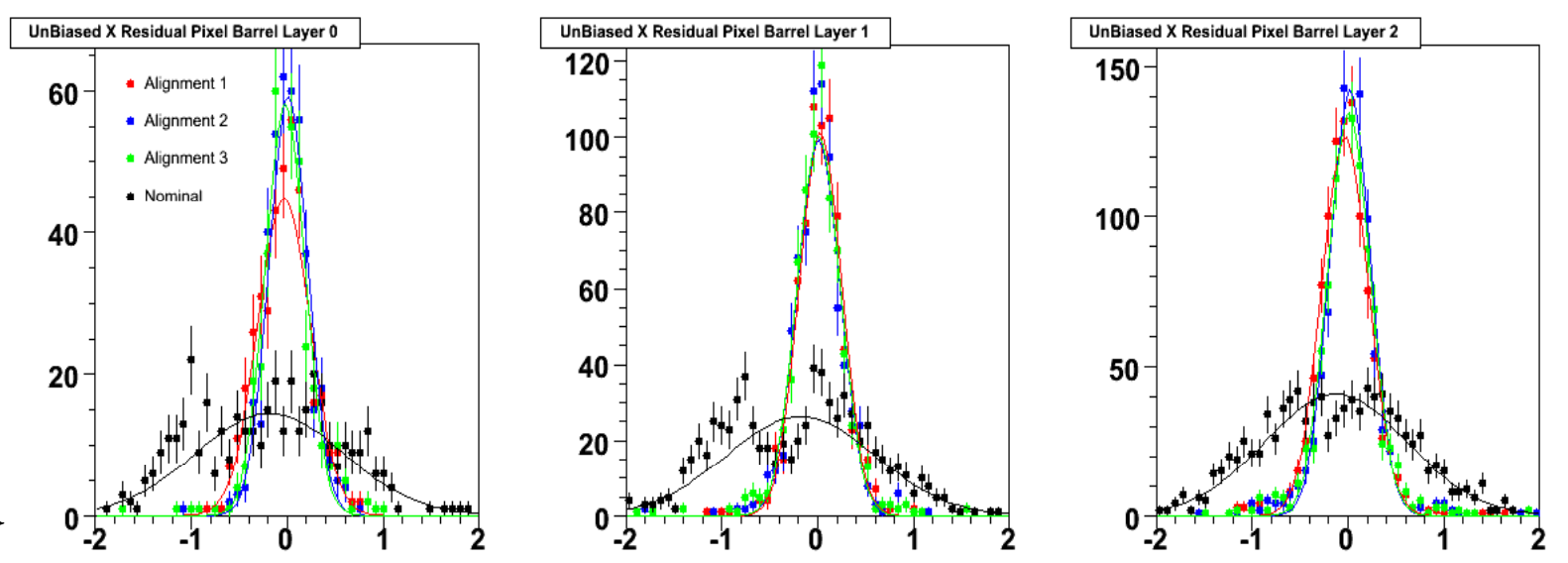

First pixel alignment from cosmics
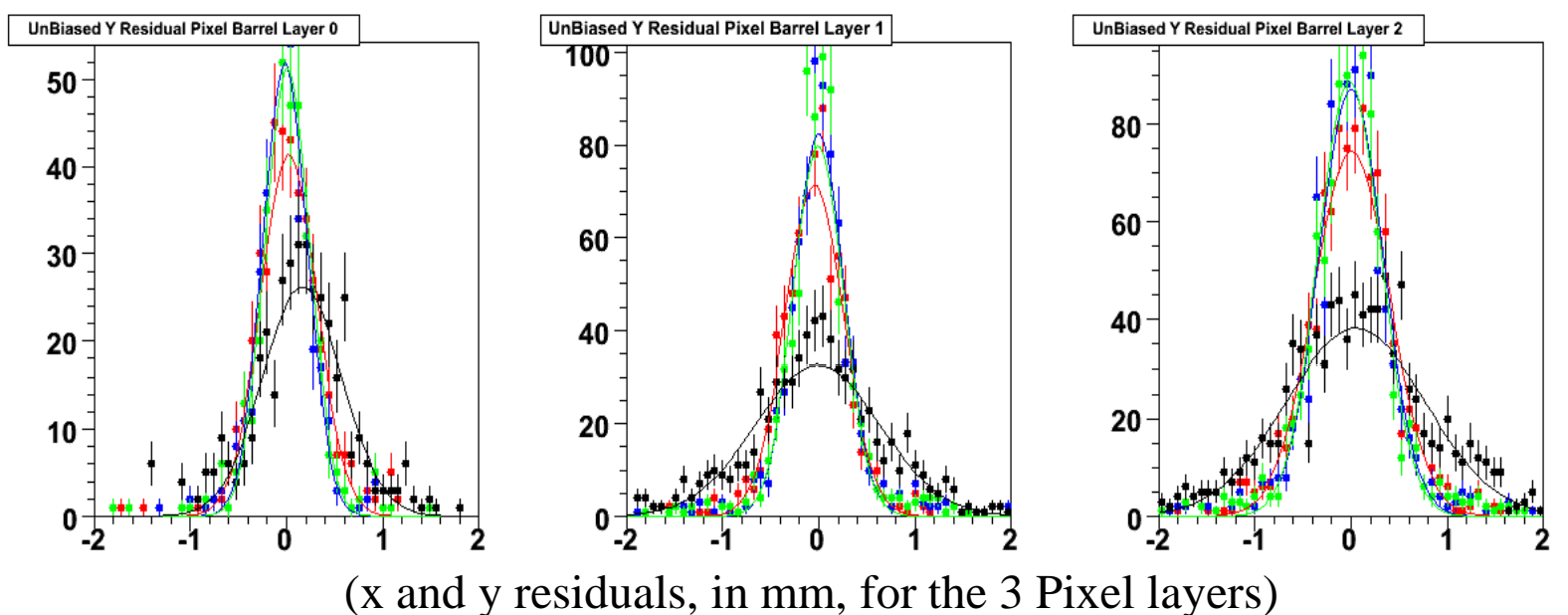

October 22, 2008

( $\mathrm{x}$ and $\mathrm{y}$ residuals, in $\mathrm{mm}$, for the 3 Pixel layers) 


\section{Commissioning Reconstruction}

- Reconstruction by standard and specialpurpose algorithms

- Inner Detector and Muon Spectrometer

- Tracks not point to nominal interaction point

- Fewer constraints for fit

- Calorimeters

- Events not synced with beam clock

- Phase for optimal filtering calculations is unknown

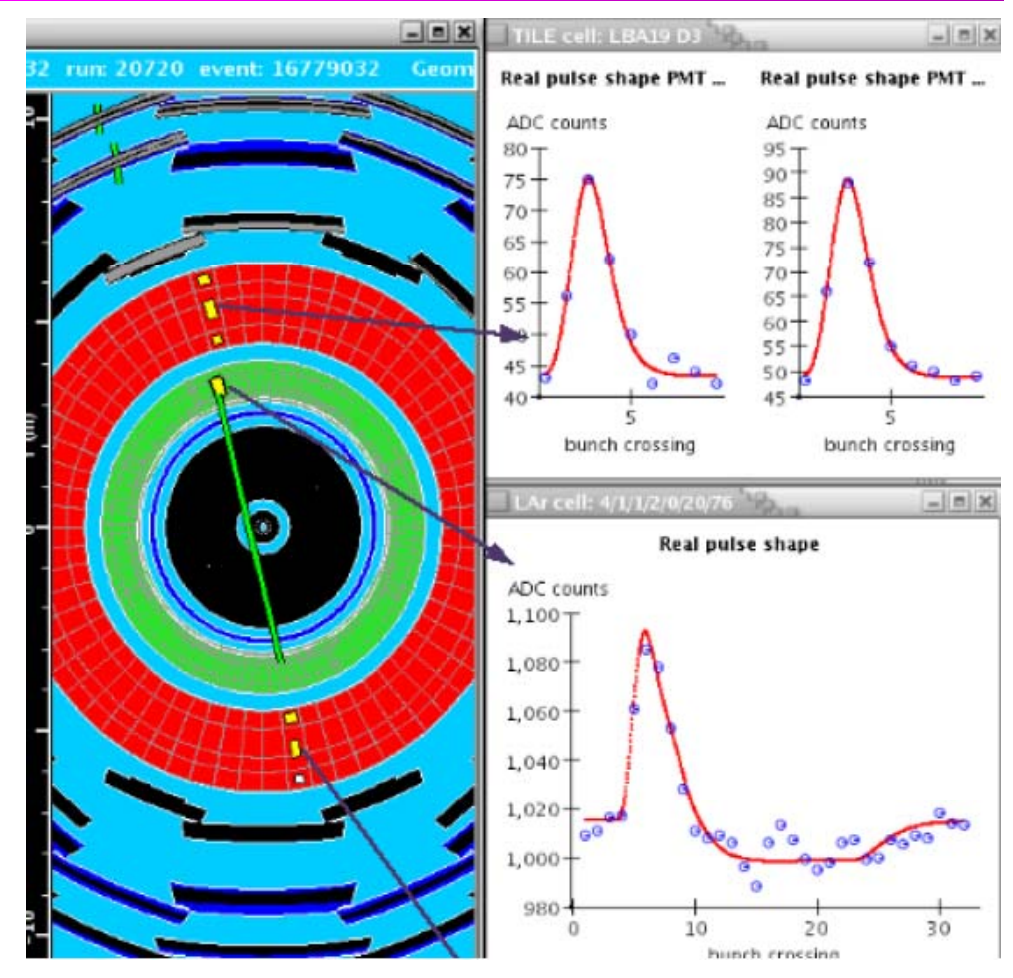

- TileCal uses specialized 3-parameter fit

- LAr uses iterative application of optimal filter coefficients

$$
E_{\text {cell }} \propto \sum a_{i} \cdot\left(s_{i}-p\right) \quad \text { Pulse samples }
$$

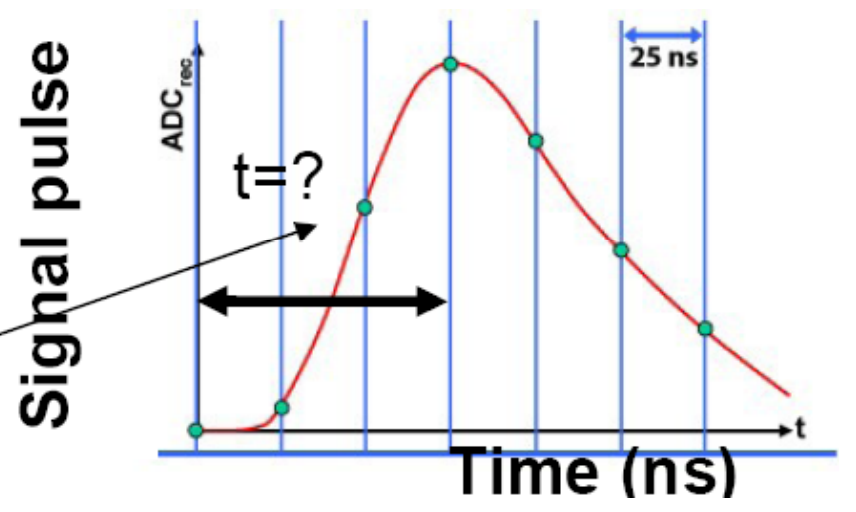




\section{Examples of Tracking}

- Tracking in various magnetic fields and combined tracking
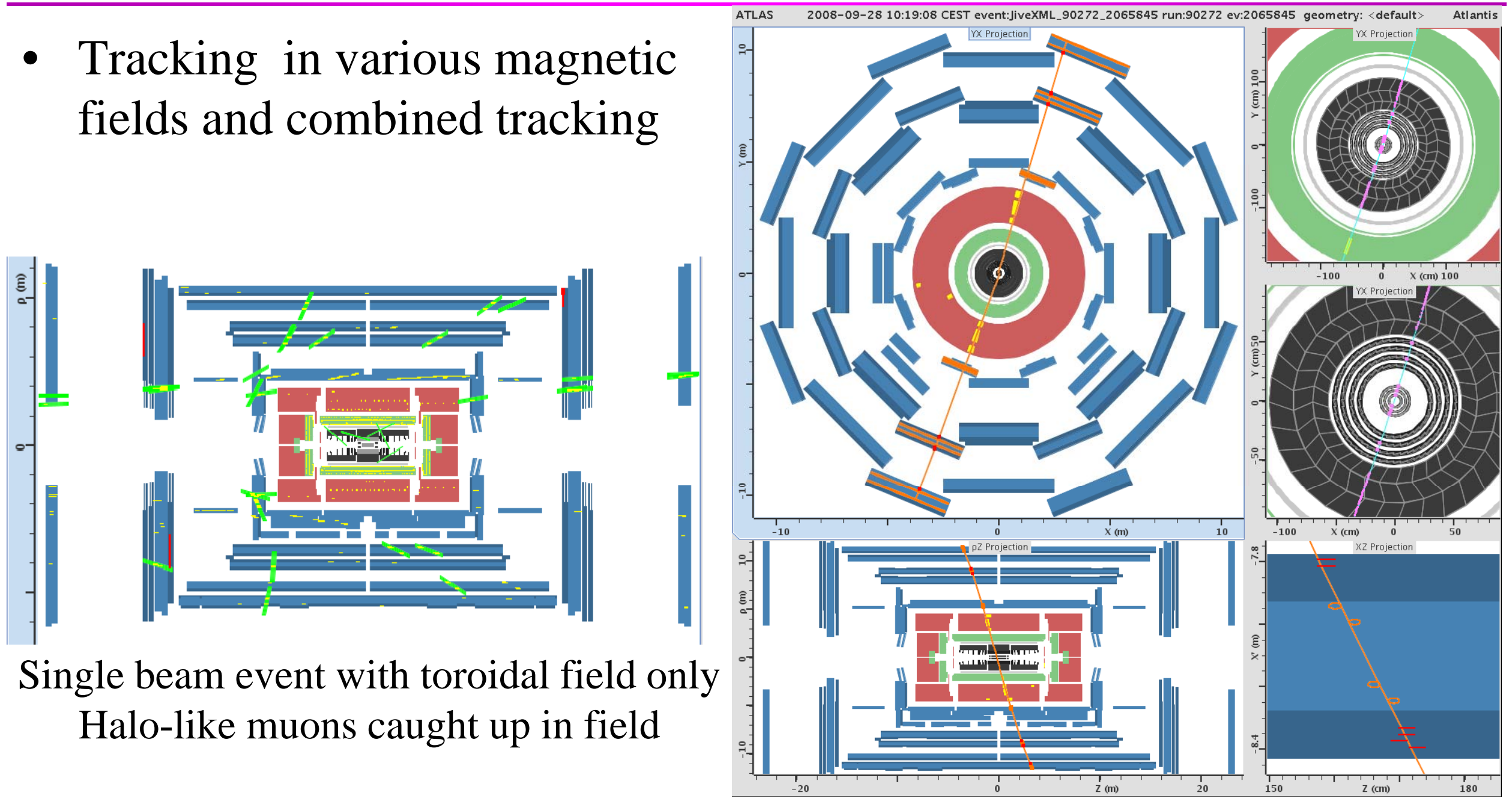

Single beam event with toroidal field only Halo-like muons caught up in field

Cosmic event with all magnetic fields, hits in all barrel detectors, and a combined track reconstructed 


\section{Detector Performance from Cosmics: Energy Reconstructed in Calorimeters}

- LAr EM

- Reconstruct E with 3x3 calorimeter cells, fit to Landau

- Eta dependence of energy agrees with MC

- Overall scale in MC has estimated 5\% systematic uncertainty

- Tile

- Muon trajectory fit from tile cells

- Energy deposited is normalized to distance traveled in tile

- Energy scale and uniformity tested to 2-3\%
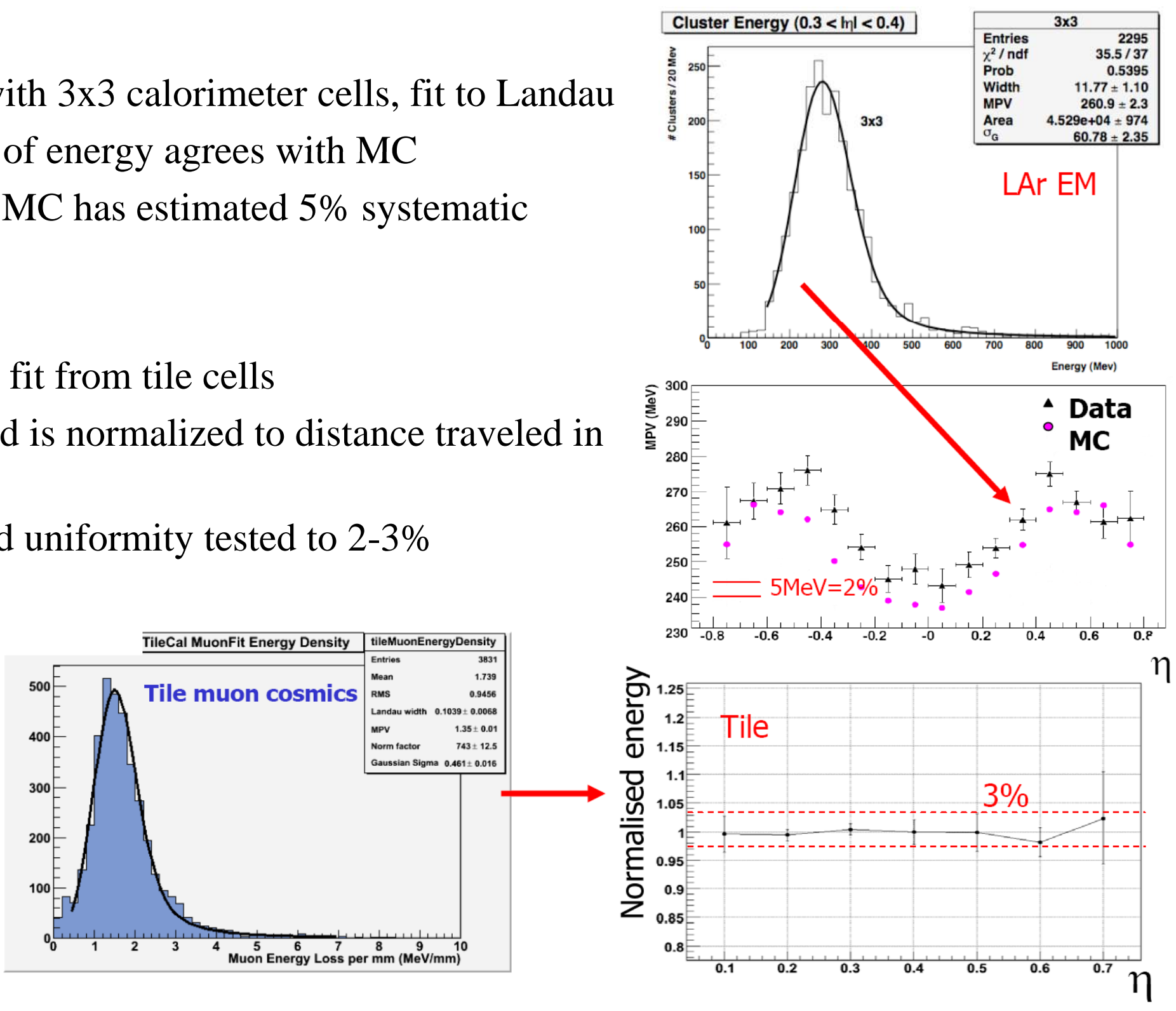


\section{Detector Performance from Cosmics: Combined Studies}

Track momentum as measured by the muon spectrometer and the inner detector. The effect of energy lost in the calorimeter is clear.

A $5 \mathrm{GeV}$ track observed in the top of the muon spectrometer loses energy in the calorimeter, and is observed e.g. as a $2 \mathrm{GeV}$ track in the inner detector.

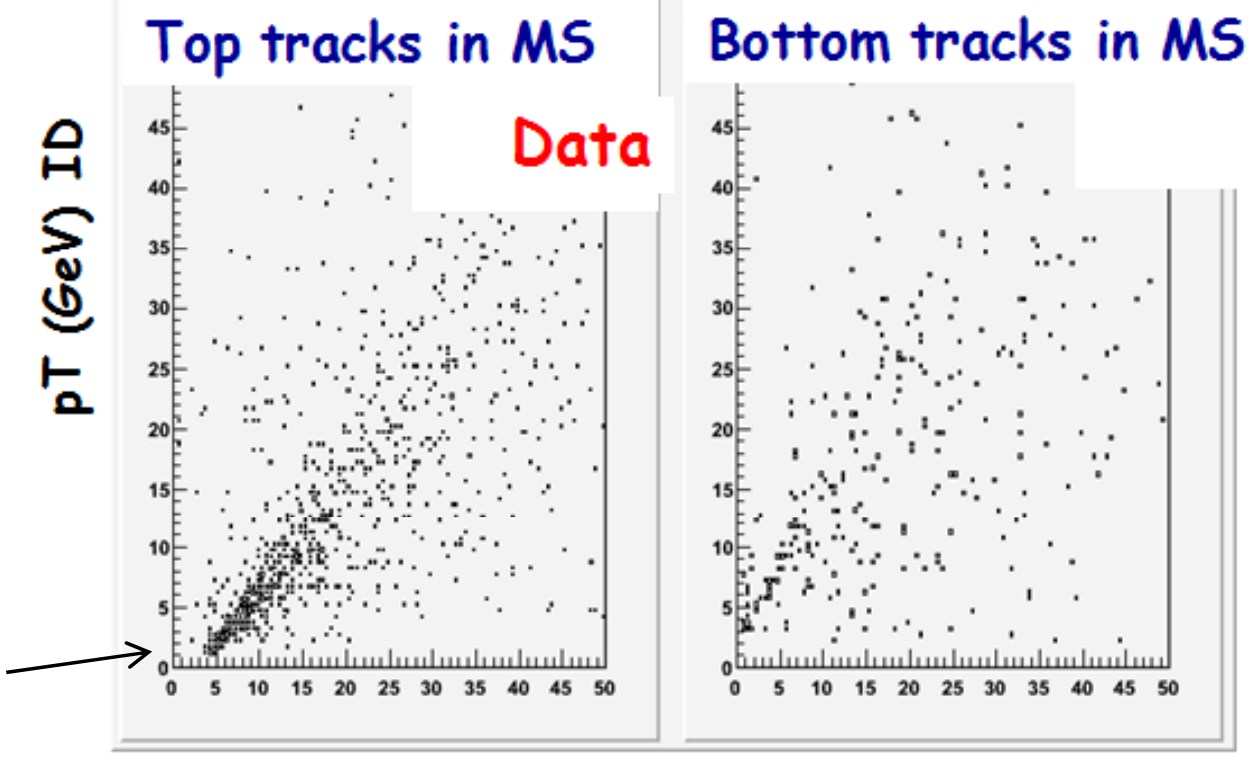

Bottom tracks in MS

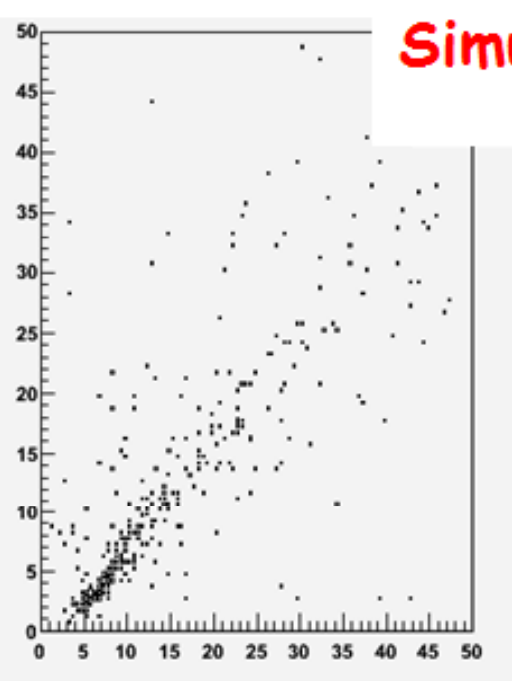

Simulation

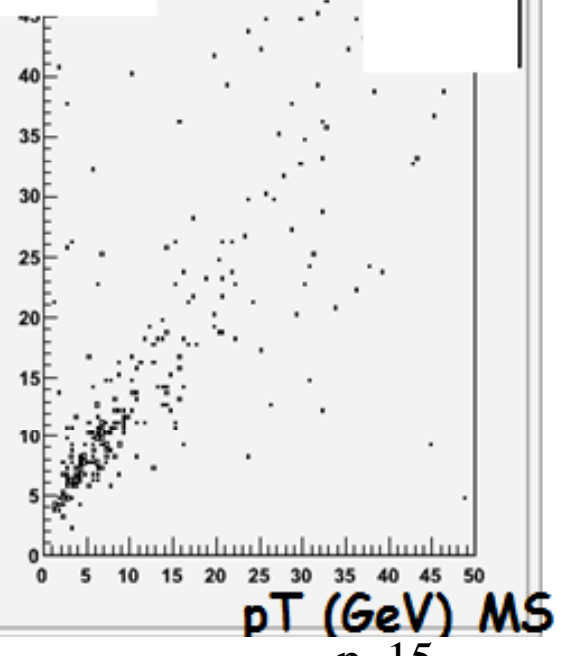

p. 15 


\section{Beam “Splash” Events}

- Beam dumped on a collimator $\sim 140 \mathrm{~m}$ from ATLAS

- Huge "splash” of energy in ATLAS

- $>1000 \mathrm{TeV}$ in calorimeters

- Hundreds of thousands of hits in muon spectrometer

- Highest energy events ATLAS will ever record?

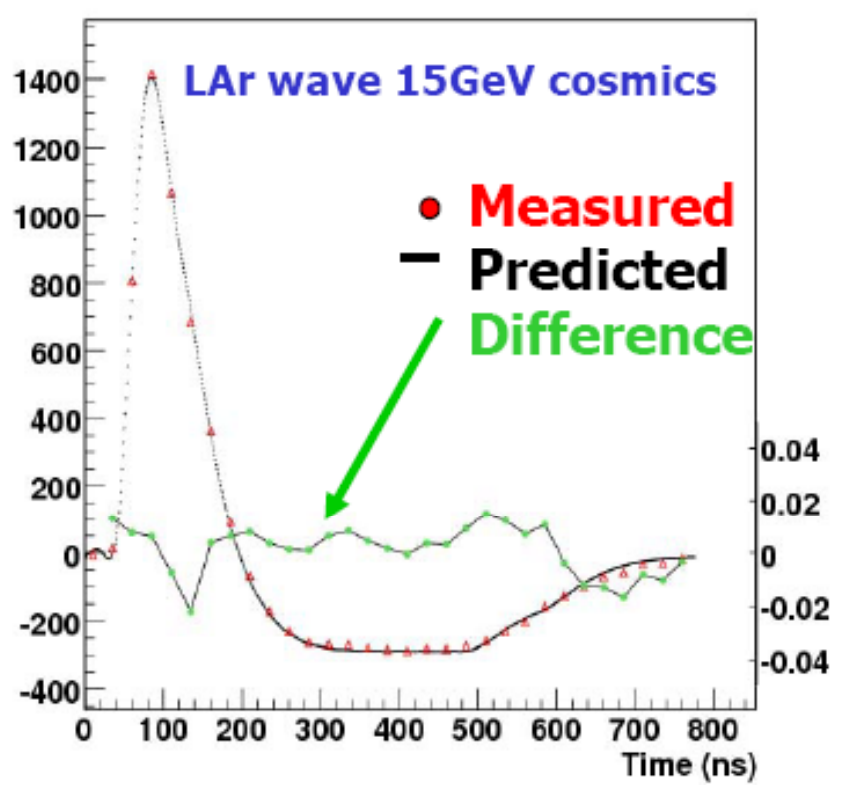

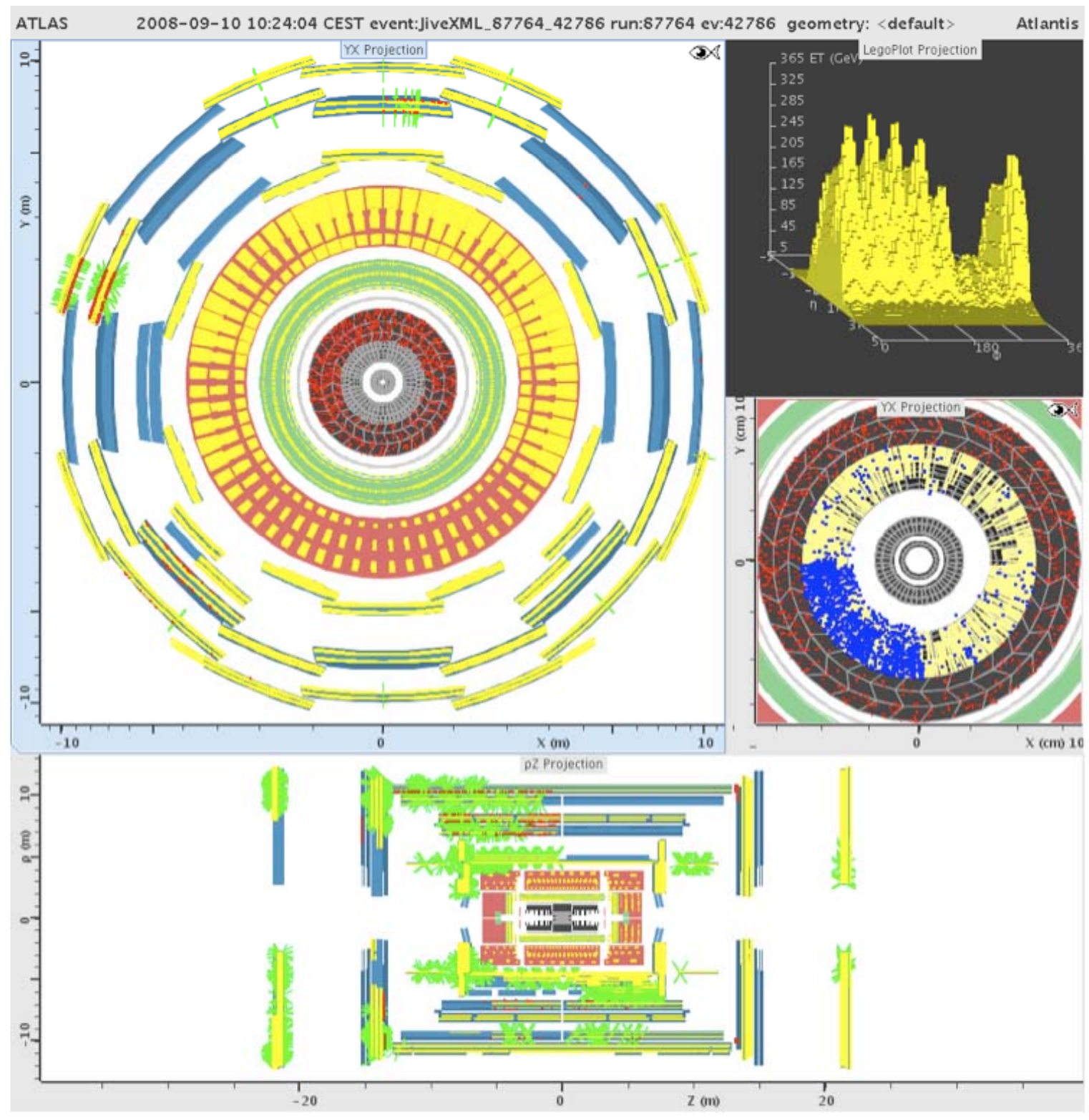




\section{Detector Commissioning: Timing with Single Beam Events}

- "Splash” events can leave signal in almost all channels in the same event

- Good source for pulse shape studies

- Excellent source for detector timing studies

- Transition Radiation Tracker timing to $1 \mathrm{~ns}$

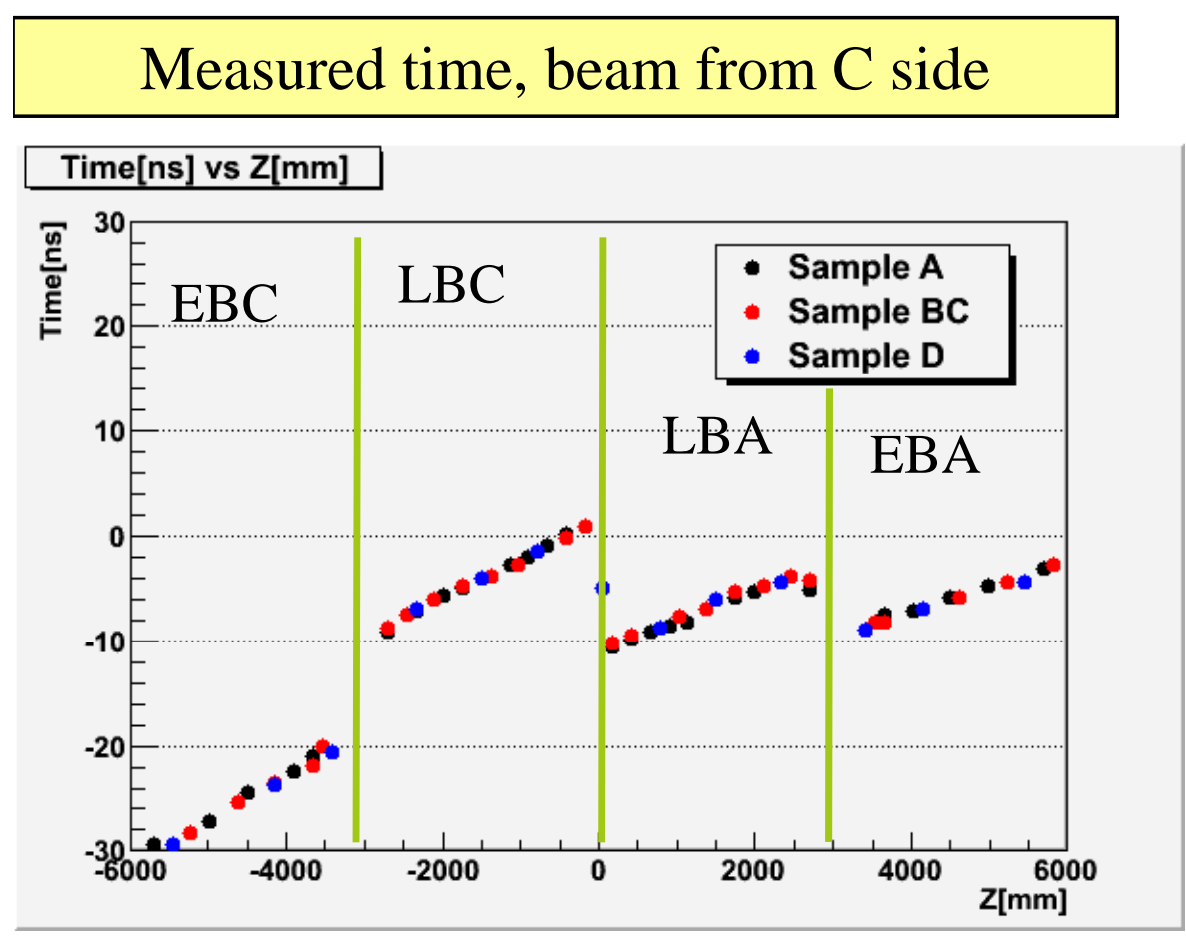

Time after TOF correction

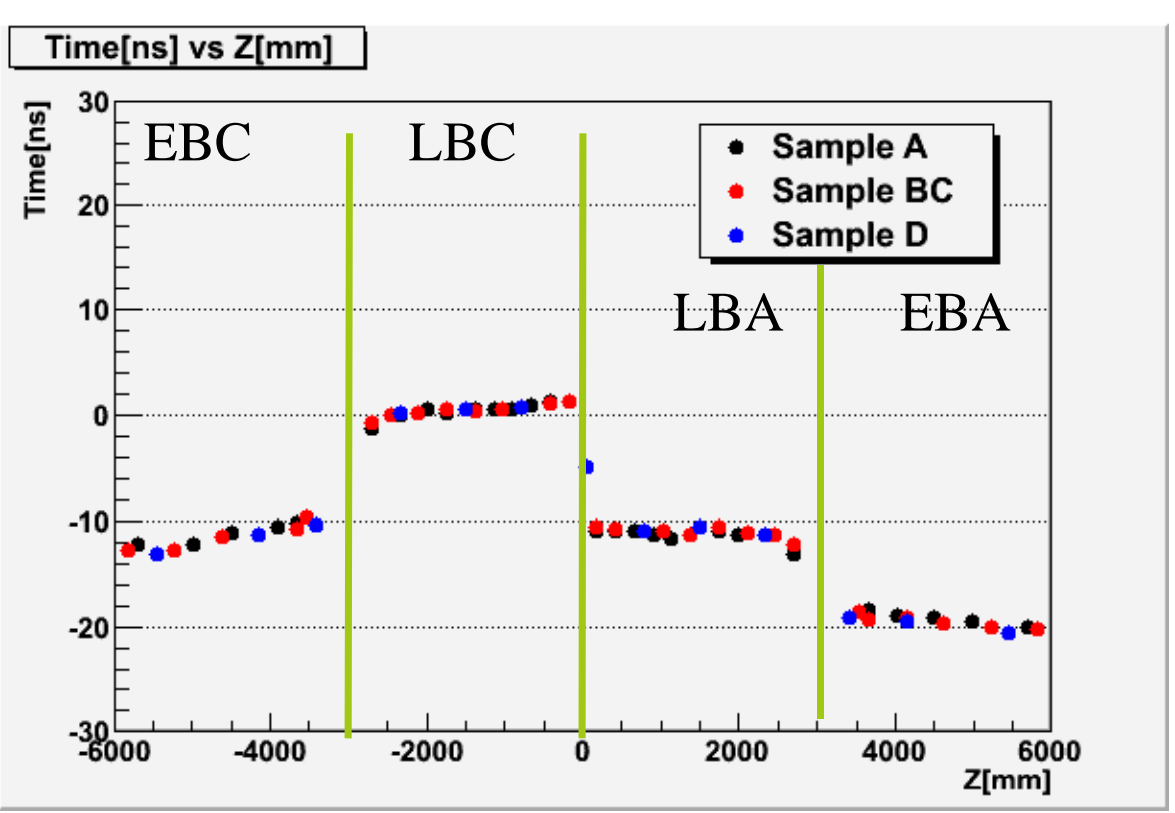

Timing measurements from 700 single beam events in Tile Calorimeter.

Cell timing within modules already calibrated. Differences between modules otherwise difficult to measure. 


\section{Software Robustness}

- Reconstruction software needs to be robust against a wide range of beam and detector conditions

- E.g. Tracking that can ignore noisy modules

- Many months of cosmics, and three days of single beam data, has provided much opportunity for debugging

No crashes on high occupancy "splash" events.

In a recent 48 hours of cosmics, 3 crashes in 16,000 reconstruction jobs.
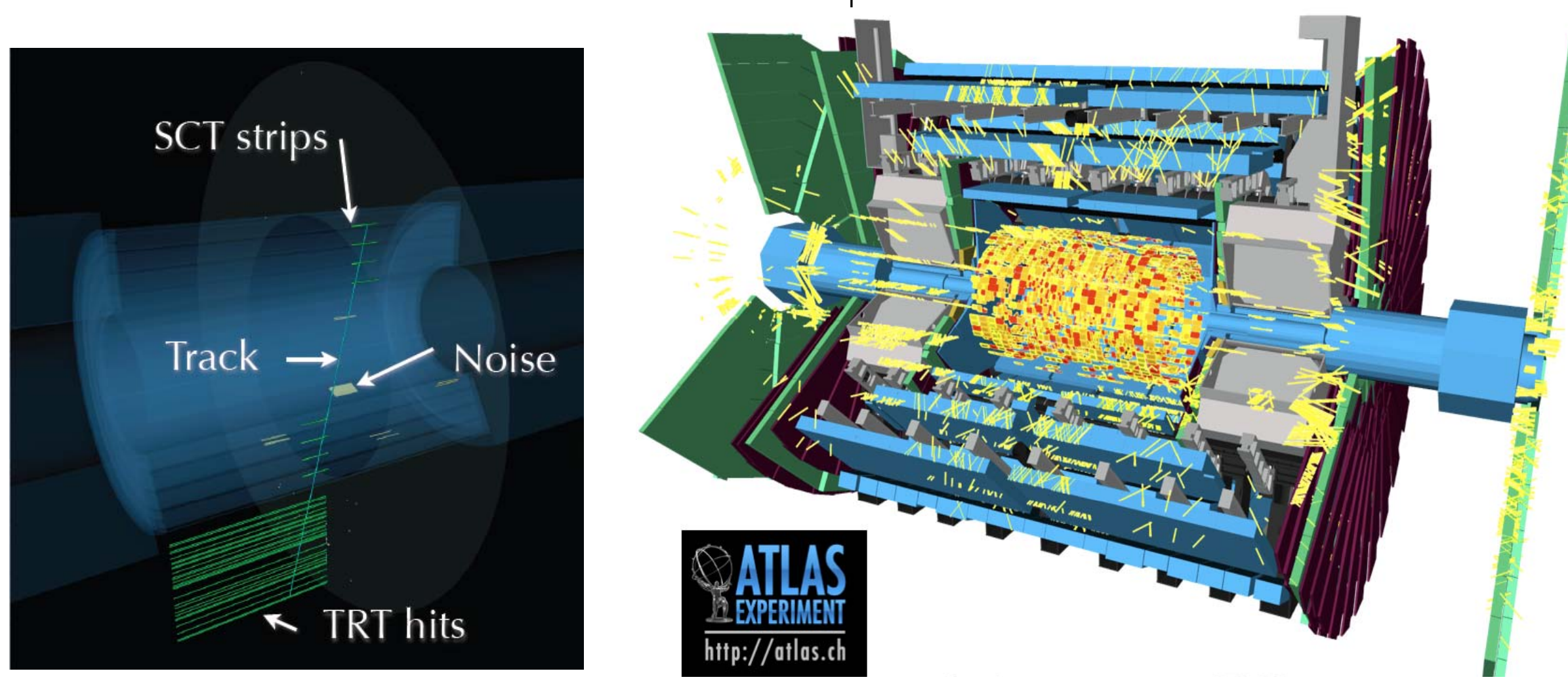


\section{Summary}

- ATLAS reconstruction software is being commissioned with first data

- Vetted with many months of cosmics

- Three days of single beam events

- Full offline reconstruction chain is in place

- High Level Trigger, Monitoring, and Event Displays

- Calibration and Alignment

- Standard and commissioning-specific algorithms

- Commissioning onsite and offsite (e.g. grid) computing infrastructure

- Operating all ATLAS sub-detectors, and all software as one system

- Integrating with the infrastructure used for MC physics studies

- Validate with real, installed detectors

- Detector performance studies aid hardware commissioning

- Looking forward to LHC collisions in 2009! 